\title{
Parálisis facial recidivante en vuelos comerciales. Reporte de un caso
}

\author{
Recurrent facial paralysis during comercial flights. Case report \\ Ignacio Mendoza A ${ }^{1}$, Ilson Sepúlveda A $^{2}$, Thomas Schmidt $\mathbf{P}^{1}$.
}

\begin{abstract}
RESUMEN
La parálisis facial asociada a cambios bruscos de presión en el oído medio es un fenómeno poco común que ha sido reportado tanto en actividades de buceo como en aviación. Se produciría una incapacidad, por parte de la tuba auditiva, de equilibrar las presiones entre el oído medio y el ambiente, lo que generaría una neuropraxia del facial en presencia de ciertas condiciones. Presentamos el caso de un paciente que desarrolló un cuadro de parálisis facial recidivante durante vuelos comerciales, se describe la evaluación realizada y la probable fisiopatología involucrada.
\end{abstract}

Palabras clave: Parálisis facial, barotrauma, baroparesia, neuropraxia, buceo, aviación.

\begin{abstract}
Facial paralysis, associated with sudden changes in pressure in the middle ear, is a rare phenomenon that has been reported in both diving and aviation. It occurs because the Eustachian tube is unable to compensate middle ear pressure changes which would lead to a facial neuropraxia in the presence of certain conditions. A case of a patient who developed recurrent facial paralysis during comercial flights. The possible involved pathophysiology is described.
\end{abstract}

Key words: Facial palsy, barotrauma, baroparesis, neuropraxia, diving, aviation.

Médico Servicio de Otorrinolaringología y Cirugía de Cabeza y Cuello, Hospital Guillermo Grant Benavente.

2 Radiólogo Maxilofacial-Cabeza y Cuello, Servicio de Otorrinolaringología, Cirugía de Cabeza y Cuello, Hospital Clínico Regional de Concepción. 


\section{INTRODUCCIÓN}

El extraño fenómeno de una parálisis facial asociado a cambios bruscos de presión en el oído medio, puede ser encontrado en la literatura también como baroparesia del facial o parálisis facial alternobárica. Esta condición ha sido reportada más frecuentemente en buceo que en aviación, dado por los mayores cambios de presión experimentados en el primero. Esta condición se produciría por la incapacidad de la tuba auditiva para ecualizar las presiones entre el oído medio y el ambiente ${ }^{1,2}$, llevando a un aumento de presión sobre el nervio facial a través de una dehiscencia en la delgada lámina ósea del canal de Falopio en su porción horizontal o timpánica, comprimiendo la vasa nervorum, generando entonces hipoxia y por consecuencia neuropraxia ${ }^{3}$. Ésta se resuelve espontáneamente en pocas horas, aunque se han reportado casos de parálisis facial más prolongados ${ }^{4}$. El primer caso publicado de parálisis facial en vuelo fue en $1986^{5}$, y hasta la fecha no más de 10 casos se pueden encontrar en la literatura. A continuación presentamos el caso de un paciente que desarrolló un cuadro de parálisis facial recurrente durante vuelos comerciales.

\section{CASO CLÍNICO}

Paciente de sexo masculino de 53 años de edad con antecedente de cirugía endoscópica de senos paranasales, por rinosinusistis crónica poliposa realizada cinco años antes y que al momento de la evaluación se encontraba sin evidencia de enfermedad tanto clínica como imagenológicamente (endoscopía y tomografía computarizada).

El paciente refería parálisis facial derecha durante vuelos comerciales (Figura 1) durante el ascenso del avión, la cual se mantenía durante todo el vuelo y desaparecía 30 a 60 minutos después de haber aterrizado. Los episodios se repitieron en cada vuelo sumando al menos unos veinte episodios.

A la otoscopía se observó tanto conducto auditivo externo como tímpanos sanos.

La audiometría resultó normal, la impedanciometría evidenció una curva "A" para el oído izquierdo y una curva "C" para el oído derecho. La prueba de función tubaria con Valsalva y degluciones de
Honjo confirmó una disfunción tubaria derecha.

El estudio de imágenes mediante Cone Beam CT de peñasco y reconstrucción del canal de Falopio demostró dehiscencia de éste en oído derecho (Figuras 2 y 3 ), sin otra anormalidad del peñasco ni del oído medio ni interno detectada. El uso de descongestionantes orales y esteroides nasales previo a los vuelos no cambió el comportamiento durante los mismos. Frente a esto, se le propone la colocación de un tubo de ventilación en el oído derecho a lo cual no accede, dada la poca duración de los episodios de la parálisis facial. Luego de tres meses de presentación del cuadro clínico, éste desaparece y no se ha repetido durante los últimos 2 años.

\section{DISCUSIÓN}

La baroparesia facial ha sido ampliamente descrita en buzos, pero en aviación son pocos los casos publicados $^{5-12}$. Sabemos que los cambios de presión generan al menos dos fenómenos muy significativos en el cuerpo humano. El primero dice relación con la disminución de la presión de oxígeno en la medida que disminuye la presión atmosférica, esto gracias a la ley de Dalton que dice que la presión total de una mezcla de gases es igual a la suma de las presiones parciales de los gases que componen

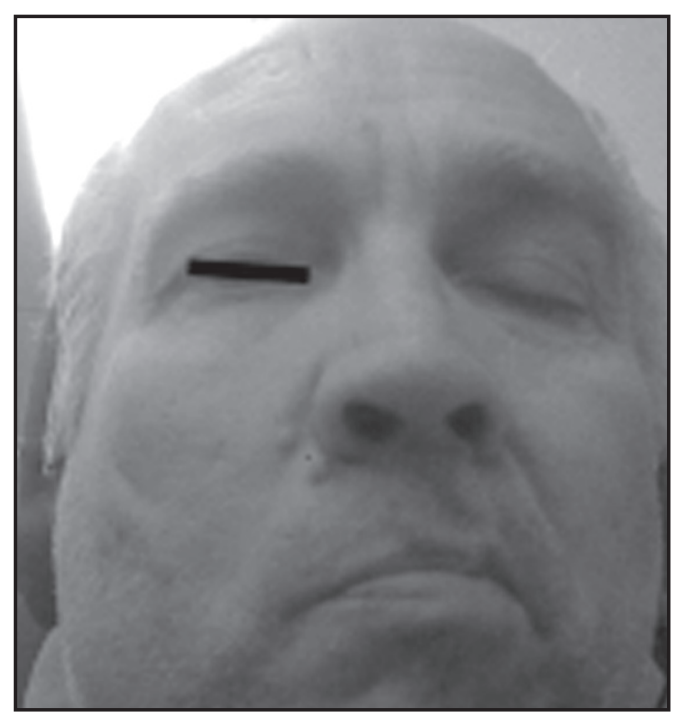

Figura 1. Foto tomada durante uno de sus vuelos. Se observa la parálisis facial derecha. 


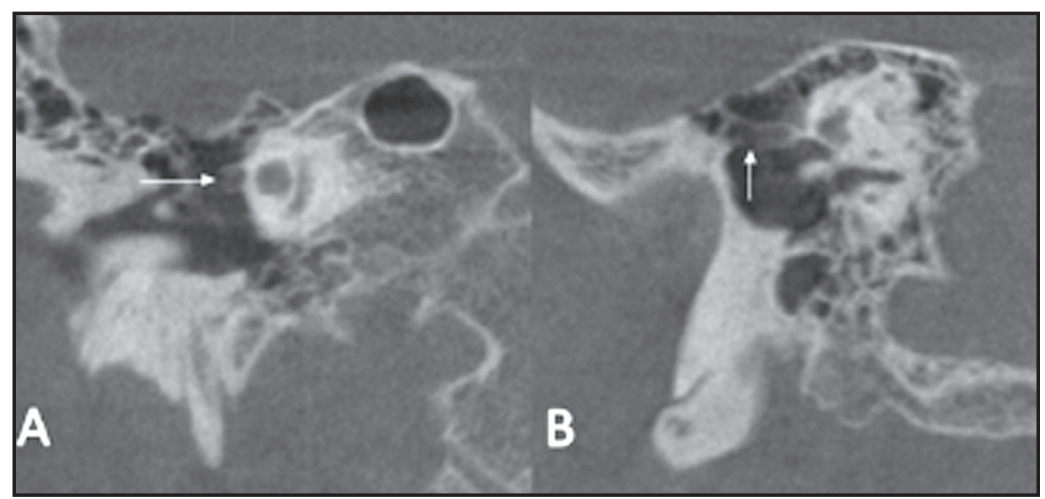

Figura 2. Cone Beam de hueso temporal derecho, vistas coronal (A) y sagital modificado (B): porción timpánica del VII par, que presenta dehiscencia ósea en su pared latero-inferior (flechas blancas).

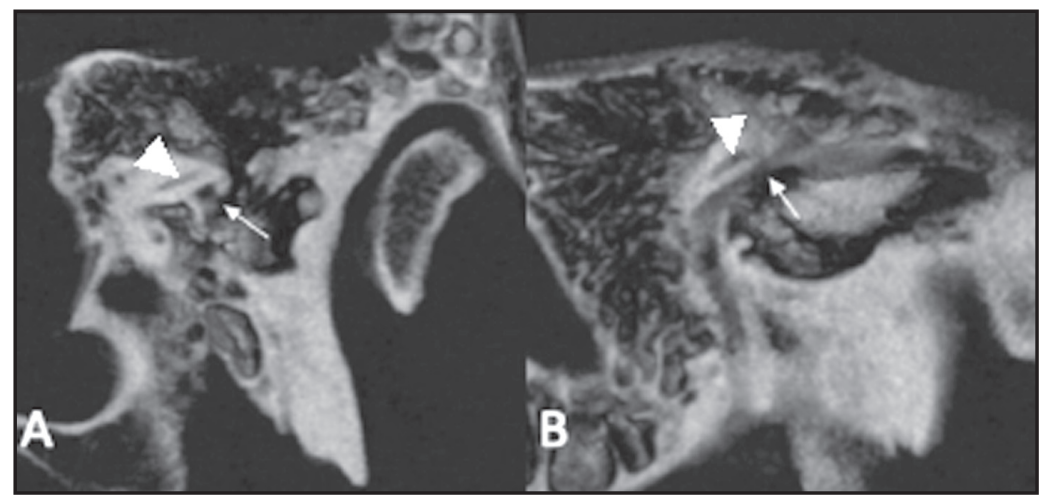

Figura 3. Reconstrucción volumétrica 3D, hueso temporal derecho, vistas coronal (A) y sagital modificado (B): dehiscencia ósea de la 2a intrapetrosa del VII par (flechas). Canal semicircular lateral (triángulos).

la mezcla. En segundo lugar, la ley de Boyle nos dice que, a una temperatura constante, el volumen de los gases aumenta en forma inversamente proporcional a la presión. Esto explica el aumento de volumen en las cavidades cerradas del organismo, como el oído medio, al ascender en altura y que es satisfactoriamente resuelto por la tuba auditiva en condiciones normales ${ }^{13}$. Un descenso de 10 metros (m) bajo el nivel del mar significa un aumento de 1 atmósfera (atm). Mucho menos dramático es en aviación, dado que un ascenso de 5.500 m recién implica una disminución de 1,5 atm. Las aeronaves comerciales vuelan a una altitud de cabina, en altitud crucero real de 35.000 pies $(\sim 10.600 \mathrm{~m})$, correspondiente a una altitud de 8.000 pies $(\sim 2.500$ $\mathrm{m})$. Esta altitud de cabina nos permite respirar sin dificultad y se denomina control isobárico, a diferencia del control de presión diferencial de los aviones de combate donde la altitud de cabina varía con el ascenso para permitir una adecuada performance del piloto frente a cambios muy bruscos de altitud. Sin embargo, en relación a la presión atmosférica, teóricamente si ascendemos desde el nivel del mar hasta la altitud crucero, la diferencia de presiones entre el oído medio y el ambiente sería de 266 $\mathrm{cmH}_{2} \mathrm{O}$, largamente por encima de la presión capilar media ${ }^{14}$ y del diferencial de presión $60 \mathrm{cmH}_{2} 0$ que se sabe produce congestión y edema del oído medio ${ }^{2,12}$. Es gracias a la presurización de cabina a 8.000 pies que se evita esto. En condiciones normales, la tuba auditiva deja escapar el gas desde el oído medio hacia la nasofaringe, pero una alteración en este mecanismo llevaría entonces a barotrauma.

En estudios llevados a cabo en cobayos se demostró que al aumentar la presión del oído medio disminuía el flujo sanguíneo del mismo $^{14}$, el cual 
sería aún menor en un nervio facial con dehiscencia de su canal, lo que sucedería en el $55 \%-59 \%$ de los huesos temporales ${ }^{1,15}$. Hay casos de paresia del trigémino en buzos por aumento de presión en el seno maxilar que respaldarían también esta teoría².

No obstante lo anterior, y dado que tanto los viajes en avión y las dehiscencias del canal del facial son frecuentes, para que ocurra una baroparesia del facial deberían coexistir ciertas circunstancias que finalmente gatillarían el fenómeno: una disfunción tubaria, un canal del facial dehisciente, hipotensión arterial y cambios bruscos de presión atmosférica.

Una hipótesis alternativa propuesta ha sido la transmisión de la presión al nervio facial con un canal no dehiscente a través de la fenestra de la cuerda del tímpano, pero no ha podido ser demostrada ${ }^{15}$.

Para el estudio de estos pacientes, la tomografía computarizada tuvo una sensibilidad de $66 \%$ y una especificidad del $84 \%-98 \%$ cuando se correlacionó con los hallazgos intraoperatorios ${ }^{16}$.

De todas formas, si sospechamos esta condición y no ha habido una resolución espontánea, la miringotomía es la conducta de elección mientras se confirma la hipótesis diagnóstica ${ }^{14}$. Para la prevención en pacientes con parálisis facial recurrente, se recomienda el uso de corticoides intranasales y antihistamínicos en pacientes alérgicos, así como de descongestionantes en pacientes con patología infecciosa de la vía aérea superior,3,8,9.

\section{BIBLIOGRAFÍA}

1. Di Martino E, Sellhaus B, Haensel J, Schlegel JG, Westhofen M, Prescher A. Fallopian canal dehiscences: a survey of clinical and anatomical findings. Eur Arch Otorhinolaryngol 2005; 262: 120-6.

2. Molvaer OI, EIDSVIK S. Facial baroparesis: a review. Undersea Biomed Res 1987;14:277-95. Ardehali MM, Yazdani N, Heidarali M. Transient facial nerve baroparesis: case report. Pak J Biol Sci 2009; 12: 476-9.

3. Hyams aF, Toynton SC, Jaramillo M, Stone LR, BRYson PJ. Facial baroparesis secondary to middle-ear over-pressure: a rare complication of scuba diving. J Laryngol Otol 2004; 118 : 721-3.

4. SilvERSTEIN H. Facial paralysis associated with air flight. Am J Otol 1986; 7: 394-5.

5. WOODHEAD CJ. Recurrent facial palsy at high altitude. J Laryngol Otol 1988; 102: 718-9.

6. Ardehal MM, Yazdani N, Heidarali M. Transient facial nerve baroparesis: case report. Pak J Biol Sci 2009; 12(5): 476-9.

7. William S B. Recurrent Facial Baroparesis Occurring on Commercial Flights in The United States. J J Neur Neurosci 2014; 1(1): 004.

8. Mirza S, Richardson R. Otic barotrauma from air travel. The Journal of Laryngology \& Otology 2005; 119: 366-70.

9. Аh-See K, Shakeel M, Mainı K. Facial paralysis during air travel: case series and literature review. The Journal of Laryngology \& Otology 2012; 126: 1063-5.

10. Rutten M, Kunst H. Facial Nerve Palsy In Aviation Facial Baroparesis. Int Adv Otol 2010; 6(2): 277-81.

11. Grossman. Facial Nerve Palsy Aboard a Commercial Aircraft. Aviation, Space, and Environmental 2004; 75(12): 1075-6.

12. Goitia Gorria A, Aguirre Ibáñez J, De Prado Jaranilla MM, Estellés Sarrió AV, Zurita A, Millán JM. Tus pacientes también vuelan. Aspectos médicos de la aviación comercial. Semergen 1999; 25: 806-17.

13. M. Motamed, H. Pau, A. Daudia, A. Narula. Recurrent facial nerve palsy on flying. The Journal of Laryngology \& Otology 2000; 114: 704-5.

14. Nagal H, Nakashima T, Suzuki T, Yanagita N. Effect of increased middle ear pressure on blood ow to the middle ear, inner ear, and facial nerve in guinea pigs. Acta Oto-Laryngol 1996; 116: 439-42.

15. BAXTer A. Dehiscence of the fallopian canal. $J$ Laryngol Otol 1971; 81: 442-9.

16. Keskin S, ÇEtIN H, Töre HG. The correlation of temporal bone CT with surgery findings in evaluation of chronic inflammatory diseases of the middle ear. Eur J Gen Med 2011; 8: 24-30.

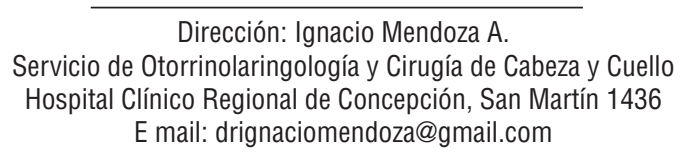

E mail: drignaciomendoza@gmail.com 